\title{
Periocular Necrotizing Fasciitis with Toxic Shock Syndrome
}

\author{
Tri Rejeki Herdiana Yasuhiro Takahashi Ma. Regina Paula Valencia \\ Marian Grace Ana-Magadia Hirohiko Kakizaki
}

Department of Oculoplastic, Orbital \& Lacrimal Surgery, Aichi Medical University Hospital, Aichi, Japan

\section{Keywords}

Periorbital necrotizing fasciitis - Toxic shock syndrome - Diagnosis - Antibiotic · Surgical debridement

\begin{abstract}
Purpose: To report a case of periocular necrotizing fasciitis with toxic shock syndrome. Methods: This is a case report of a previously healthy 69-year-old woman with left preseptal eyelid infection that spread rapidly and deteriorated into necrosis of the eyelid with toxic shock syndrome. She was admitted to intensive care unit for hemodynamic stabilization. Results: Intravenous antibiotic and high-dose immunoglobulin were administered followed by surgical debridement. Rehabilitative eyelid reconstruction was performed after acute episode, resulting in patient satisfaction in relation to periocular function and appearance. Conclusion: We reported a case of periocular necrotizing fasciitis with toxic shock syndrome that necessitated early aggressive medical treatment and adequate surgical intervention to decrease morbidity and mortality. A high level of suspicion of periocular necrotizing fasciitis is necessary to make a prompt diagnosis.




\section{Introduction}

Necrotizing fasciitis (NF) is a rare, severe, and devastating infection of the subcutaneous soft tissue and superficial fascia with secondary necrosis in the overlying skin [1,2]. NF usually affects the limbs or the abdominal wall. Periorbital involvement is rare with an associated mortality of $14.4 \%$, which is low compared to the other parts of the body [2,3]. Similar to orbital or preseptal cellulitis, periocular NF presents with eyelid swelling, erythema, and periorbital pain. However, the complication of periocular NF is more severe as it may lead to toxic shock syndrome and death [4]. Early diagnosis and immediate treatment is essential to decrease mortality. We report a case of periorbital NF with toxic shock syndrome that was managed via a multidisciplinary approach.

\section{Case Report}

A 69-year-old female presented with a 5-day history of fever and left eyelid swelling. This was accompanied by partial gray-tan discoloration of the involved eyelid of over 3 days' duration. Pertinent past medical history includes aesthetic upper and lower blepharoplasty on both sides.

On initial examination, there was severe left eyelid swelling (Fig. 1a), which made the rest of the ocular examination difficult. The patient also had fever at $39.2^{\circ} \mathrm{C}$ during initial examination. Serologic examination revealed $\mathrm{HbA1c}$ of $6.3 \%$ and her laboratory risk indicator for necrotizing fasciitis (LRINEC) score [5] was 2 points. Orbital computed tomography (CT) showed left soft tissue swelling of the periocular and temporal regions (Fig. 1b). Diagnosis of periorbital cellulitis was made and she was given with $2 \mathrm{~g} /$ day of intravenous flomoxef sodium. Blood, urine, and eyelid swab samples were obtained for bacterial culture. She was then referred to an infectious disease specialist and endocrinologist for co-management.

Five hours after admission, the patient developed cold sweats and abdominal pain. Systolic blood pressure dropped from 120 to $50 \mathrm{~mm} \mathrm{Hg}$. LRINEC score increased to 8 points, and eyelid necrosis was noted (Fig. 1c). Clinical diagnosis of NF with toxic shock syndrome was made and the patient was admitted to intensive care unit for hemodynamic support. The patient was treated with $1.8 \mathrm{~g}$ /day of intravenous clindamycin, $6 \mathrm{~g}$ /day of ampicillin, and 1.2 $\mathrm{g} /$ day of vancomycin. She was also given $5 \mathrm{~g}$ /day intravenous immunoglobulin for 3 days. Emergency debridement for necrotic skin and subcutaneous tissue was performed until healthy viable tissue was seen around the wound (Fig. 1d). Histopathology of the debrided tissues showed massive infiltration of neutrophils between surface to muscular layers with necrosis (Fig. 1e). Two days after debridement, blood culture and eyelid swab revealed Streptococcus pyogenes infection. Intravenous vancomycin was discontinued. Seven days after debridement, white blood cell count and C-reactive protein were within normal limits, which prompted discontinuation of intravenous clindamycin. Twelve days after debridement, adverse drug reaction was observed. As $\beta$-lactam allergy was confirmed by a dermatologist, the patient was then shifted to oral levofloxacin. Eyelid reconstruction was performed 14 days after debridement with combination of skin graft and local skin flaps. Thereafter, suspicious adverse drug reaction was observed 17 days after debridement. As drug eruption from levofloxacin was initially suspected, this was then discontinued. Subsequent investigation by a dermatologist revealed that the drug eruption was not due to levofloxacin. The rest of the hospital stay was unremarkable and she was sent home and advised regular follow-up. 
On the 13-month follow-up, the patient could voluntarily open her left eye and had normal visual function and mild lagophthalmos (Fig. 1f). The patient was satisfied with her appearance.

\section{Discussion}

We have reported a case of periorbital NF with toxic shock syndrome. The patient was managed by a multidisciplinary team composed of an oculoplastic specialist, intensivist, infection disease specialist, endocrinologist, and dermatologist.

Risk factors for periocular NF include surgical trauma, the use of immunomodulatory medicine, diabetes mellitus, collagen vascular disease, and alcoholism [1]. Our patient had an elevated HbA1c value, which could have been a risk factor for the development of periocular NF. The history of cosmetic upper and lower eyelid surgery was also identified as a possible risk factor in this case. However, fulminating infection usually develops within a few hours to 5 days after a traumatic event [6-8] and a 3-year interval from the surgery may be far-fetched to be considered as a risk factor.

Laboratory and imaging studies are essential diagnostic modalities for periocular NF [2]. Orbital CT in this patient showed soft tissue swelling in the periocular and temporal regions, which is compatible with periocular NF in the early disease process. The LRINEC score is also a useful tool for early detection of patients with $\mathrm{NF}[5,9]$. Our patient had a LRINEC score of 8 , which was strongly predictive of this disease.

Our patient was treated with a combination of intravenous antibiotic and high-dose immunoglobulin. Clindamycin is known to be effective against Streptococcus species by inhibiting the synthesis of both M protein and streptococcal pyogenic exotoxins $[1,10]$. High-dose immunoglobulin serves as an adjunct for neutralization of superantigen activity and aids in antibiotic perfusion $[1,10]$.

Adequate surgical debridement of necrotic tissue decreases the bacterial load and the subsequent production of collagenase, hyaluronidase, and M proteins. This step is crucial to decrease morbidity and mortality $[1,10,11]$. Emergency debridement of necrotic tissue may have aided in reducing the white blood cell count and C-reactive protein levels to normal range after 7 days. Debridement of devitalized tissue until healthy viable tissue was seen around the wound was essential to avoid recurrence of eyelid necrosis. Eyelid reconstruction using skin graft and local skin flap was done 14 days after the acute episode, giving the patient satisfaction with her periocular function and appearance.

In conclusion, when presented with a case of a suspicious periocular NF, early diagnosis and aggressive management through a multidisciplinary approach is critical for limiting the complications of NF and preventing further morbidity and mortality.

\section{Statement of Ethics}

This retrospective observational case report has adhered to the tenets of the 1964 Declaration of Helsinki. Institutional review board approval and the patient's written informed consent for the publication of this report have been obtained. 


\section{Disclosure Statement}

There are no competing interests.

\section{Author Contributions}

No one contributed to the work that did not meet our authorship criteria.

\section{References}

1 Amrith S, Hosdurga Pai V, Ling WW. Periorbital necrotizing fasciitis- a review. Acta Ophthalmol. 2013 Nov;91(7):596-603.

2 Lazzeri D, Lazzeri S, Figus M, Tascini C, Bocci G, Colizzi L et al. Periorbital necrotising fasciitis. Br J Ophthalmol. 2010 Dec;94(12):1577-85.

3 Rajak SN, Figueira EC, Haridas AS, Satchi K, Uddin JM, McNab AA et al. Periocular necrotising fasciitis: a multicentre case series. Br J Ophthalmol. 2016 Nov;100(11):1517-20.

4 Shield DR, Servat J, Paul S, Turbin RE, Moreau A, de la Garza A et al. Periocular necrotizing fasciitis causing blindness. JAMA Ophthalmol. 2013 Sep;131(9):1225-7.

5 Wong CH, Khin LW, Heng KS, Tan KC, Low CO. The LRINEC (Laboratory Risk Indicator for Necrotizing Fasciitis) score: a tool for distinguishing necrotizing fasciitis from other soft tissue infections. Crit Care Med. 2004 Jul;32(7):1535-41.

6 Kronish JW, McLeish WM. Eyelid necrosis and periorbital necrotizing fasciitis. Report of a case and review of the literature. Ophthalmology. 1991 Jan;98(1):92-8.

7 Jordan DR, Mawn L, Marshall DH. Necrotizing fasciitis caused by group A streptococcus infection after laser blepharoplasty. Am J Ophthalmol. 1998 Feb;125(2):265-6.

8 Goldberg RA, Li TG. Postoperative infection with group A beta-hemolytic Streptococcus after blepharoplasty. Am J Ophthalmol. 2002 Dec;134(6):908-10.

9 Hodgins N, Damkat-Thomas L, Shamsian N, Yew P, Lewis H, Khan K. Analysis of the increasing prevalence of necrotising fasciitis referrals to a regional plastic surgery unit: a retrospective case series. J Plast Reconstr Aesthet Surg. 2015 Mar;68(3):304-11.

10 Seal DV. Necrotizing fasciitis. Curr Opin Infect Dis. 2001 Apr;14(2):127-32.

11 Wilson MP, Schneir AB. A case of necrotizing fasciitis with a LRINEC score of zero: clinical suspicion should trump scoring systems. J Emerg Med. 2013 May;44(5):928-31. 


\section{Case Reports in Ophthalmology}

www.karger.com/cop

Herdiana et al.: Periocular Necrotizing Fasciitis
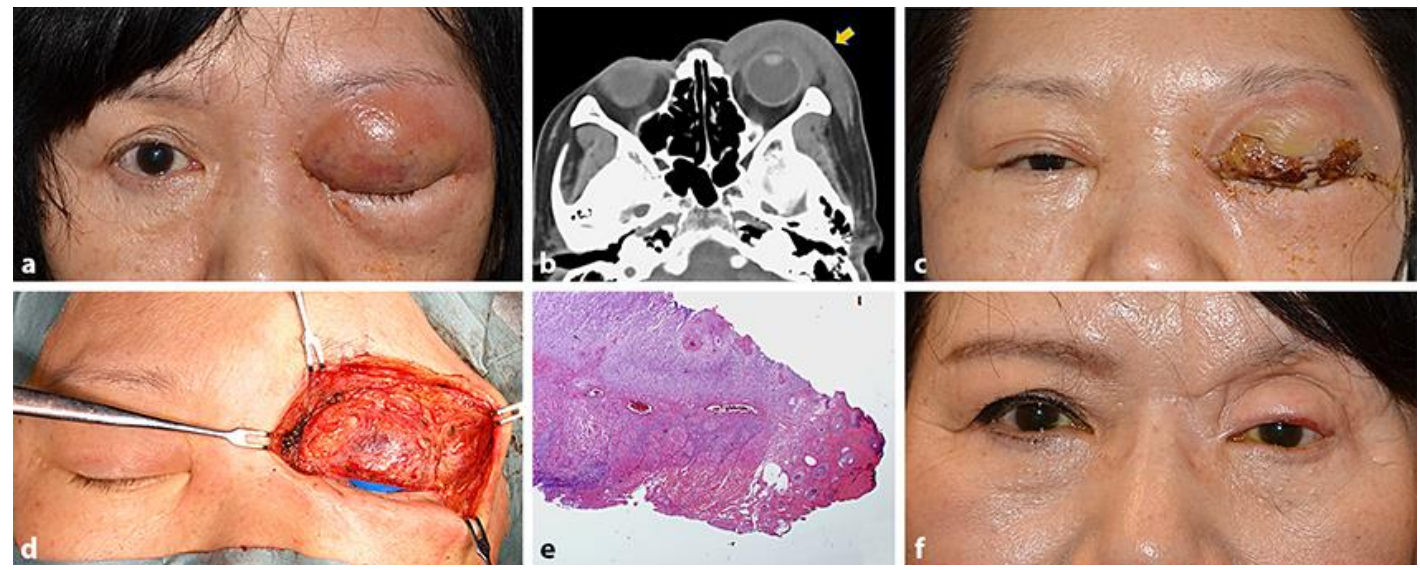

Fig. 1. a Patient's face photo taken on the first examination. The left eyelid is swollen with partial gray-tan discoloration. b Axial computed tomographic image taken on the first examination showing marked soft tissue swelling of the periocular and temporal regions. $c$ Patient's face photo taken $5 \mathrm{~h}$ after the first examination showing left eyelid necrosis. $\mathbf{d}$ Photo taken during emergency debridement of necrotic skin and subcutaneous tissue. Healthy viable tissue is present all around the wound. e Photo of the histopathology of the debrided tissues showing massive infiltration of neutrophils between surface to muscular layers with necrosis (hematoxylin and eosin stain; magnification, $\times 20$ ). $f$ Patient's face photo taken at the 13month follow-up. 